\title{
Patients with Type 2 Diabetes Mellitus failing on oral agents and starting once daily insulin regimen; a small randomized study investigating effects of adding vildagliptin
}

Wendela Lucia de Ranitz-Greven ${ }^{*}$, Joline Wilhelma Johanna Beulens², Lette Birgit Elisabeth Anne Hoeks ${ }^{1}$, Gerdien Belle-van Meerkerk', Douwe Hedde Biesma ${ }^{1}$ and Harold Wessel de Valk

\begin{abstract}
Background: The addition of a DDP4-inhibitor to existing insulin therapy reduces HbA1c. However, no data exist about the addition of these agents at the beginning of insulin treatment in type 2 diabetes while this could especially be interesting because it is during this period that considerable residual beta cell function is still present. The benefit of such a strategy could be a lower insulin dose required for glycemic control. The hypothesis of our study was that adding a DPP4-inhibitor at the beginning of insulin treatment could lead to less exogenous insulin requirement, a reduction of hyperinsulinemia and side effects (hypoglycemia and weight gain), less glucose variability and improvement of insulin and glucagon dynamics during a mixed meal test.

Results: In this small clinical trial (trial registration NTR2022) 9 patients were randomized to receive vildagliptin and 6 to receive placebo in addition to start of once daily insulin treatment. Unfortunately, due to a difficult inclusion, the preset sample size of 40 patients could not be met. Median units of insulin at the end of the study was $47 \mathrm{U}$ in the placebo group and $34 \mathrm{U}$ in the vildagliptin group. Median glycemic variability (SD) at the end of study was 2.1 in the placebo group and 1.5 in the vildagliptin group. Median weight gain at the end of study was $3 \mathrm{~kg}$ in the placebo and $0.5 \mathrm{~kg}$ in the vildagliptin group. Occurrence of hypoglycemia was low in both groups. Insulin, C-peptide, glucose and glucagon levels were comparable during mixed meal tests.

Conclusions: This small randomized study did not have sufficient power to detect effects of the addition of vildagliptin to the start of once daily long-acting insulin. However in our opinion adding a DPP4-inhibitor, especially in this group remains a very interesting approach. This study could be used as a guidance for larger studies that are required to investigate the effects of this intervention on insulin requirements, glycemic variability, hypoglycemia and weight gain.
\end{abstract}

Keywords: Clinical trial, DPP4-inhibitor, Insulin, Therapy Vildagliptin

\footnotetext{
* Correspondence: w.l.greven@umcutrecht.nl

${ }^{1}$ Department of Internal Medicine, University Medical Centre Utrecht,

Huispostnummer, Postbus 85500 3508, Utrecht, GA F02-126, The Netherlands

Full list of author information is available at the end of the article
} 


\section{Background}

Due to the progressive nature of the disease, most patients with type 2 diabetes mellitus (DM) ultimately fail on oral glucose-lowering drugs and therefore require insulin therapy [1,2]. Often considerable doses of insulin are needed and weight gain and hypoglycemia can occur [1]. The dipeptidyl peptidase-4 inhibitor (DPP4-inhibitor) vildagliptin is an oral glucose-lowering drug that leads to glucose-dependent insulin secretion and improvement of alpha cell function [3]. A reduction in glycated haemoglobin (HbA1c) and less hypoglycemia was shown in a previous study adding vildagliptin on top off an existing insulin regimen [4]. Multiple randomizedcontrolled trials investigating the effect of DPP4inhibitor added to existing insulin have been performed since [4-14]; however, no data exist about vildagliptin use at the start of insulin treatment in patients with Type 2 DM. Adding a DPP4-inhibitor at the beginning of insulin treatment could especially be interesting because it is during this period that considerable residual beta cell function is still present. The benefit of such a strategy could be a lower insulin dose necessary for glycemic control. To study this, we titrated insulin in all patients to the best achievable degree of glycemic control. Therefore, we did not use HbAlc as the primary end-point, as most previous studies on DPP4-inhibitors and insulin did, but the required insulin dose instead.

The aim of the study was to investigate whether or not the addition of a DPP4-inhibitor to start of insulin treatment could lead to lower exogenous insulin requirements together with lower glucose variability, less weight gain, less hypoglycemia, and improved insulin and glucagon dynamics after a mixed meal test through improvement in alpha and beta cell function.

\section{Results}

In this study 19 patients were screened and 4 excluded (reasons for exclusion in (Additional file 1). Nine patients were randomized to receive vildagliptin and 6 patients to placebo (Additional file 1). This study was designed to include 40 patients in 1 year. Since inclusion rate was much slower than expected, the inclusion period was extended by more than a year. However, the number of 40 patients could still not be met and inclusion was therefore terminated after 15 patients.

Table 1 shows the baseline characteristics, which were comparable in both treatment groups.

Results are summarized in Table 2. Median number of units of insulin in the placebo group was 47 and in the vildagliptin group 34 . In both groups compliance was high and HbA1c decrease was comparable. Median glycemic variability (SD) in the placebo group was 1.8 at the beginning of the study and 2.1 at the end, in the vildagliptin group glycemic variability was 1.7 and 1.5 respectively. Median weight increase at the end of the study was $3 \mathrm{~kg}$ in the placebo and $0.5 \mathrm{~kg}$ in the vildagliptin group. Occurrence of hypoglycemia was low and comparable in both groups. Median systolic blood pressure change in the vildagliptin group was +2.5 and $-3 \mathrm{mmHg}$ in the placebo group. LDL and SAF only showed very minor changes.

Comparable levels of glucose, insulin, C-peptide and glucagon were observed in both groups after mixed meal tests (Additional file 2: Figure S2A-D). Median values of area under curve (AUC) of glucose and glucagon were 1704 (1439-2279) and 5100 (3030-7620) in the vildagliptin group and 1773 (1439-1931) and 5310 (3525-10785) in the placebo group. Median levels of AUC of insulin and Cpeptide were 5295 (1695-24705) and 343050 (106395$963000)$ in the vildagliptin group and 3795 (1710-7095) and 199703 (134550-281250) in the placebo group. Adverse events were reported more often in the placebo group. These consisted of flu like symptoms (3 patients, all in the placebo group), tiredness (1 vildagliptin, 1 placebo), toothache/parodontitis (1 vildagliptin, 1 placebo), a cold (1 vildagliptin), headache and diarrhea (1vildagliptin), reversible increase in gGT (1 placebo, max $165 \mathrm{U} / \mathrm{l}$ (normal value: 0-40 U/L)), sensation of tingling in the left arm, which was reversible during the study (1 placebo), mild orthostatic symptoms (1 vildagliptin). None of these events were considered to be severe or related to the study medication.

\section{Table 1 Base-line characteristics}

\begin{tabular}{lll}
\hline & Placebo & Vildagliptin \\
\hline $\mathrm{N}$ & 6 & 9 \\
Age (years) & $60(39-67)$ & $64(42-67)$ \\
$\mathrm{BMI}(\mathrm{kg} / \mathrm{m} 2)$ & $32(26-35)$ & $32(27-34)$ \\
Female $(\%)$ & $17 \%$ & $22 \%$ \\
Duration DM (years) & $5.5(1-23)$ & $6.0(2-15)$
\end{tabular}

Prior medication

metformin/SU/TZD/other (\%)

$100 / 67 / 0 / 33 \% \quad 100 / 89 / 11 / 22 \%$

Diabetic complications

any microvascular complication (\%) $\quad 17 \% \quad 33 \%$

any macrovascular complication (\%) $\quad 0 \% \quad 33 \%$

BP (syst/diast in $\mathrm{mmHg}$ ) 128/78 126/73

$\mathrm{HbA1c}$ at start of the trial $(\mathrm{mmol} / \mathrm{mol}) \quad 64(53-74) \quad 62(57-73)$

(\%) $\quad 8.0(7.0-8.9) \quad 7.8(7.4-8.8)$

SD of glucose values (variability) at start $\quad 1.8(1.0-2.7) \quad 1.7(1.2-2.6)$ of trial

All values are in median (range) or percentages.

Any micro- or macrovascular complication was defined as the percentage of patients who had one or more complications as judged clinically by the investigator at the moment of inclusion.

Abbreviations: $\mathrm{BMI}=$ body mass index, $\mathrm{DM}=$ diabetes mellitus, $\mathrm{SU}=$ sulfonylurea $\mathrm{TZD}=$ thiazolidinediones, $\mathrm{BP}=$ blood pressure, $\mathrm{HbA} 1 \mathrm{c}=$ glycated haemoglobin, $\mathrm{SD}=$ standard deviation. 
Table 2 Results

\begin{tabular}{|c|c|c|}
\hline & Placebo & Vildagliptin \\
\hline \multicolumn{3}{|l|}{ Primary endpoint } \\
\hline Units insulin & $47(16-62)$ & $34(12-62)$ \\
\hline \multicolumn{3}{|l|}{ Secondary endpoints } \\
\hline Glycemic variability end of study (SD) & $2.1(1.1-2.8)$ & $1.5(1.0-3.6)$ \\
\hline Change in weight (kg) & $3(-2.5-5.5)$ & $0.5(-2.6-4)$ \\
\hline $\begin{array}{l}\text { Hypoglycemia during the study } \\
\text { (nr per pat) }\end{array}$ & $1.5(0-5)$ & $1.0(0-8)$ \\
\hline \multicolumn{3}{|l|}{ Change in blood-pressure ( $\mathrm{mmHg})$} \\
\hline Systolic & $-3(-12-6)$ & $2.5(-14-10)$ \\
\hline Diastolic & $-1.5(-9-3)$ & $-0.5(-6-11)$ \\
\hline Change in LDL (mmol/L) & $0(-1-0.6)$ & $-0.3(-1.4-0)$ \\
\hline Change in SAF (skin AGEs) (AU) & $0.1(-0.4-0.8)$ & $0.15(-0.4-0.6)$ \\
\hline \multicolumn{3}{|l|}{ Safety } \\
\hline $\begin{array}{l}\text { Patients with one or more hypoglycemia } \\
\text { (\%) }\end{array}$ & $67 \%$ & $78 \%$ \\
\hline Patients with severe hypoglycemia (\%) & $0 \%$ & $0 \%$ \\
\hline Patients with one or more AE (\%) & $100 \%$ & $44 \%$ \\
\hline Patients with one or more SAE (\%) & $0 \%$ & $0 \%$ \\
\hline \multicolumn{3}{|l|}{ Other } \\
\hline Delta HbA1c (mmol/mol) & $-6.5(-18-7)$ & $-6(-25-4)$ \\
\hline (\%) & $-0.6(-1.6-0.6)$ & $-0.5(-2.3-0.3)$ \\
\hline Compliance (\% of tablets taken) & $96 \%$ & $98 \%$ \\
\hline
\end{tabular}

All values are in median (range) or percentages.

Compliance: $\%$ of tablets taken during the whole study period.

Delta HbA1c: HbA1c end-begin, -6 means a decrease of 6 points in $\mathrm{HbA} 1 \mathrm{c}$ during the study, a number without - means an increase.

Because of the small sample size no $p$-values are shown.

Abbreviations; $L D L=$ low-density lipoprotein, SAF = skin autofluorescence,

AGEs $=$ advanced glycation end products, $A U=$ arbitrary units, $A E=$ adverse event, $\mathrm{SAE}=$ serious adverse event, $\mathrm{HbA} 1 \mathrm{c}=$ glycated hemoglobin .

\section{Discussion}

This is the first study investigating the addition of a DPP4inhibitor to the start of once-daily long-acting insulin in insulin-naïve patients. Although this study was set up as a double-blind parallel-arm placebo-controlled trial, the sample size is limited and therefore we cannot make conclusions about the effect of addition of vildagliptin on units of insulin at the end of the study, variability, weight, hypoglycemia or response of insulin, C-peptide or glucagon after a mixed meal test.

This important limitation is due to difficulties in patient recruitment. In the Netherlands, most patients starting with once-daily insulin are treated by a general practitioner and not in an (academic) hospital, where this study was performed. Despite extensive collaboration with many general practitioners and hospitals in the region, patients were still difficult to recruit. The small sample size is therefore too small to have statistical power to confirm or reject the null hypothesis.
However, given the working mechanism of DPP4inihibitors and our small sample size, we think it would be very interesting to investigate our hypothesis in a larger study. Our small study could potentially serve as a guidance for such larger studies. From our data it can be derived that a repeat study would need approximately 46 patients per group. This is based on a median units of insulin of 40 (SD 17) and 10 units of insulin decrease, which would be clinically significant (and 25\% is comparable to the difference in median units of insulin in our study of $28 \%$ ). This sample size calculation must be considered as a rough estimation, given the small sample size and not-normal distribution of our main end-point, but at this moment the best estimation available.

Besides the smaller sample size our study differs in two major design aspects from previous randomized-controlled trials. First, previous trials investigated the effect of add-on DPP4-inhibition therapy to existing insulin regimen [4-14], whereas we investigated the addition of vildagliptin to the start of insulin therapy in our study. We hypothesized that adding a DPP4-inhibitor to start of insulin treatment could lead to less exogenous insulin requirements. This could lead to a reduction of hyperinsulinemia, which is thought to have atherogenic and mitogenic effects [15]. Moreover, lower insulin use could reduce side effects of insulin treatment such as hypoglycemia and weight gain [1]. The addition of a DPP4-inhibitor to an existing insulin treatment without the intention to change the insulin regimen as previous trials did does not reflect clinical practice in which physicians will choose to alter insulin schedules. Only in one randomized trial the insulin dose was changed as a goal in one arm (insulin-increasing arm) and compared to the addition of sitagliptin to existing insulin regimen. In that trial, a difference of $25 \%$ in insulin dose was described between the insulinincreasing and the insulin-sitagliptin group [11] together with a more pronounced HbA1c decrease in the sitagliptin group. The $25 \%$ decrease in units of insulin found in that study is comparable to the magnitude (28\%) we found.

Second, we used a different end-point compared to previous trials. In randomized-controlled trials about the effect of the addition of DPP4-inhibitors to insulin thus far, HbA1c or glycemic control were used as primary end-point [4-14]. We chose required units of insulin since, in our study, we added vildagliptin to the start of insulin and we aimed at the best glycemic control with insulin glargine in all patients.

Because a lower insulin dose may lead to less side effects, we also investigated these as secondary end-points. The first was hypoglycemia. With improvement of glycemic control, the incidence of hypoglycemic episodes was expected to increase. This was indeed observed in a study using sitagliptin [14]. In contrast, a study by 
Fonseca et al. showed less hypoglycemia together with a decrease in HbA1c when vildagliptin was added to an insulin regimen [4], although this could not be confirmed in a study by Kothny et al., using the same DPP4-inhibitor [10]. Less hypoglycemia, as shown in the study by Fonseca, could be a result of an improved alpha cell function, which has been described not only postprandial (reduction in glucagon) but also during the reaction after induced hypoglycemia when a slightly increased increment of glucagon was seen [3]. In our study occurrence of hypoglycemia was low in both groups. The second secondary end-point studied was weight. In contrast to GLP1-analoga, DPP4-inhibitors do not show a large effect on weight [16]. Differences in body weight were not shown in previous randomized-controlled trials with DPP4 inhibitors in combination with insulin [4-14], except for one study which compared insulin-increasing therapy with addition of sitagliptin to existing insulin regimen [9]. The design of that study most resembled ours, and it also found a difference in required units of insulin between the groups. Furthermore, that study showed less weight increase in the DPP4 addition group (between-group difference $1.7 \mathrm{~kg}$ in favour of the DPP4-group, which is in concordance in magnitude with our findings (vildagliptin group (+0.5 kg), placebo group (+3 kg).

We also studied additional end-points, including glucose variability. We hypothesize that since variability is determined by hyperglycemic and hypoglycemic excursions, effects on alpha cell function could result in less glucose variability. A decrease in postprandial glucagon could reduce hyperglycemic excursions, while an increase in glucagon response following a hypoglycemia could reduce a hypoglycemic excursion. A decrease in variability has been reported before from single-arm trials, investigating the effect of addition of a DPP4-inhibitor to insulin $[17,18]$. In our study, median glycemic variability (SD) at the end of the study was 2.1 in the placebo group and 1.5 in the vildagliptin group. We evaluated responses of insulin, Cpeptide and glucagon during a mixed meal test, which were comparable in our study. It is difficult to assess insulin and glucagon dynamics in patients taking insulin [14]. All patients were on stable insulin dose, titrated at the fasting glucose levels and all patients were fasting. However, since exogenous insulin was present at time of testing because all patients took their glargine at bedtime the day before the testing, we cannot exclude that measurements are confounded by exogenous insulin. Since GLP1 could also have an effect on cardiovascular parameters, these were also included as an endpoint [19]. Levels for blood pressure, LDL and skin autofluorescence were comparable.

\section{Conclusions}

This small randomized study did not have sufficient power to detect effects of the addition of vildagliptin to the beginning of once daily long-acting insulin. However in our opinion adding a DPP4-inhibitor, especially in this group remains a very interesting approach. This study could be used as a guidance for larger studies to investigate the effects of this intervention on insulin requirements, glycemic variability, hypoglycemia and weight gain.

\section{Methods \\ Design}

This study was set up as a double-blind parallel-arm placebo-controlled randomized monocenter trial (16 weeks) comparing the effects of adding vildagliptin or placebo to the start of insulin in patients with type 2 diabetes mellitus. Patients were included from December 2009 - May 2012.

\section{Patients}

Patients who were scheduled by their treating physician to start once-daily long-acting insulin were eligible. Inclusion criteria were: Type 2 DM, failing on maximally tolerated oral-glucose-lowering medication, BMI 25-35, HbA1c $53-75 \mathrm{mmol} / \mathrm{mol}(7.0-9.0 \%)$ and age $25-75$ years. Exclusion criteria were: pregnant women or women in the fertile period of life without adequate birth-control, type I DM, or another type of DM (for example pancreatic injury, prednisone induced), acute metabolic diabetic complications during the last 6 months, severe cardiac (left ventricle ejection fraction $(\mathrm{LVEF})<30 \%$ ) or (a history of) hepatic failure (transaminases $>3$ times elevated), or renal impairment (creatinine clearance $<50 \mathrm{ml} / \mathrm{min}$ ).

\section{Randomization}

Patients were randomized to receive vildagliptin $(50 \mathrm{mg}$ twice daily) or matching placebo (twice daily). To prevent confounding by BMI, patients were randomized after stratification for BMI (using two different randomized lists for BMI $25-30 \mathrm{~kg} / \mathrm{m}^{2}$ or BMI $30-35 \mathrm{~kg} / \mathrm{m}^{2}$ ). Consecutive patients were allocated to the two different groups using two computer-generated randomized lists (block size 4), which were stored in sealed envelopes. Randomization and distribution of blinded study medication was performed by a person not related to the study (pharmacy). Patients and care providers were unaware of the randomization code.

\section{Treatment}

Besides the study medication, all patients started with once daily long-acting insulin glargine at bedtime in combination with a fixed dose of metformin (twice daily $850 \mathrm{mg}$ ). Other glucose lowering drugs were terminated. The combination with metformin was used since this is standardized approach in Dutch clinical practice. The dose of insulin glargine was protocolized and titrated based on daily fasting blood glucose measurements of the patients and an algorithm comparable to the one published by Davies et al. [20]. In short, all patients 
started with 8 units insulin glargine at bedtime and performed daily fasting glucose measurements. The insulin dose was increased based on the mean of the last glucose measurements. This was performed twice a week for the first 3 weeks and once a week during the remainder of the study. The insulin dose was raised as follows: mean glucose 5.5-6.7 mmol/L: raise of 0-2 units, 6.7$7.8 \mathrm{mmol} / \mathrm{L}: 2$ units, $7.8-10 \mathrm{mmol} / \mathrm{L}: 4$ units, $>10 \mathrm{mmol} /$ L: 6-8 units increase. The dose was only raised in the absence of hypoglycemia.

\section{Outcomes}

Primary outcome: units of insulin at the end of the study.

Secondary outcomes: 1) glycemic variability estimated by the standard deviation (SD) of 48 hours glucose values at the end of the study as measured by three days blinded continuous glucose measurement (CGM)) [21] 2) change in weight 3) hypoglycemia (defined as the total number of hypoglycemia during the study period (any glucose selfmeasurement $<4.0 \mathrm{mmol} / \mathrm{L}$ or symptoms which the patients recognises as hypoglycemia and clinically judged by the investigator as hypoglycemia)) 4) severe hypoglycemia (defined by help needed from others, seizure, or coma) 5) alpha and beta cell function (between group comparison of glucose, insulin, C-peptide and glucagon levels, measured after standardised meal) 6) cardiovascular analysis; change in blood pressure (mean ambulant 24 hour arterial pressure) and change in plasma lipids 7) change in skin Advanced glycation end products (AGEs) (measured as skin-autofluorescence by the AGE-reader) [22,23].

Adverse events (AE): any (worsening of an) undesirable sign, symptom, or medical condition that was noted by the patient occurring after starting the study drug even if the event was not considered to be related to the study drug.

Serious adverse events (SAE): AE judged as medically significant, requiring in-patient hospitalization, prolongation of existing hospitalization, or resulting in persistent or significant disability or incapacity or are life-threatening or fatal.

\section{Study procedures}

Age, BMI, duration of DM, medication use, history of diabetic complications, blood pressure, prior hypoglycemia (defined as symptomatic hypoglycemia in the past year), HbA1c, glucose variability and laboratory values were recorded at baseline. Patients were seen every 4 weeks in the out-patient clinic in our academic hospital and with telephone consultation every week. Drug accountability and laboratory measurements were performed at 8 and 16 weeks. CGM and ambulant blood pressure measurement were repeated at the end of the study. At the end of the study a standardised mixed meal test (MMT) was performed. Patients arrived fasting at the outpatient clinic and took their medication (metformin and study medication) at $\mathrm{t}=0$, after blood samples were drawn as a baseline $(t=0)$. At $t=30$ blood samples were drawn again and they consumed a standardized breakfast within 15 minutes (containing $522 \mathrm{Kcal} ; 27$ gr protein, 60 gr carbohydrates, 18 gr fat). Blood samples for glucose, insulin, C-peptide and glucagon were then drawn at $t=45,60,90,120,150$ and 210 minutes. Insulin and C-peptide were measured using an electrochemiluminescence immunoassay on the Modular E170 (Roche Diagnostics GmbH, D-68298 Mannheim, Germany). Finally glucagon was measured with a competitive radioimmunoassay (Glucagon KGND1, Siemens Healthcare Diagnostics Inc, Los Angeles, USA).

The study was conducted using Good Clinical Practice according to the declaration of Helsinki. The protocol was approved by the ethics committee of the UMCUtrecht and all patients provided written informed consent. This investigator-driven study was designed, performed and analysed by the researchers of the UMCUtrecht. Novartis provided study medication and a research grant. The protocol of this trial was published before start of the trial at www.trialregister.nl (NTR2022).

\section{Sample size calculation}

The group size was based on the primary study parameter: the absolute difference in daily insulin dose of long-acting insulin between the two groups at the end of the trial. A previous study in patients starting on once-daily insulin showed a mean insulin glargine dose of $57+/-15$ units [24]. A meaningful decrease would be a difference of at least 14 units. A similar decrease (25\%) in insulin dose has been observed when adding metformin to existing insulin treatment [25]. With a beta of 0.2 and a one-sided alpha of 0.05 , the minimum number of patients in each group is 15. To correct for unforeseen circumstances, we planned to include 20 patients in each group.

\section{Statistics}

Results are presented as median and range, categorical parameters as percentages. Areas under the curve of glucose, insulin, C-peptide and glucagon were calculated using the trapezoidal method. Since we did not meet our intended sample size results are presented in a pure descriptive way.

\section{Additional files}

Additional file 1: Figure S1. Randomization scheme of the trial.

Additional file 2: Figure S2. Glucose, insulin, C-peptide and glucagon

levels after mixed meal test.

\section{Abbreviations}

AE: Adverse event; AGEs: Advanced glycation end products; AUC: Area under curve; BMl: Body mass index; CGM: Continuous glucose measurement;

DM: Diabetes mellitus; DPP4-inhibitor: Dipeptidyl peptidase-4 inhibitor; 
GLP1: Glucagon-like peptide-1; HbA1c: Glycated hemoglobin; LVEF: Left ventricle ejection fraction; MMT: Mixed meal test; SAE: Serious adverse event; SD: Standard deviation.

\section{Competing interests}

This investigator-driven study was designed, performed and analysed by the researchers of the UMCUtrecht. Novartis provided study medication and a research grant. The authors declare that they have no competing interests.

\section{Authors' contributions}

WR participated in the design, conduct and data collection of the study, as well as the analysis and manuscript preparation. JB, DB and HdV participated in the design and analysis of the study, as well as manuscript preparation. $\mathrm{LH}$ and GB participated in the conduct and data collection of the study as well as manuscript preparation. All authors read and approved the final manuscript.

\section{Acknowledgements}

We thank all general practitioners and medical specialists for referring patients for our study.

\section{Funding}

Study medication and a research grant were provided by Novartis b.v.

\section{Author details}

${ }^{1}$ Department of Internal Medicine, University Medical Centre Utrecht, Huispostnummer, Postbus 85500 3508, Utrecht, GA F02-126, The Netherlands. ${ }^{2}$ Julius Centre for Health Sciences and Primary care, University Medical Centre Utrecht, Utrecht, The Netherlands.

Received: 1 April 2014 Accepted: 18 August 2014

Published: 29 August 2014

\section{References}

1. U.K. Prospective Diabetes Study Group: U.K. prospective diabetes study 16. Overview of 6 years' therapy of type II diabetes: a progressive disease. Diabetes 1995, 44:1249-1258.

2. Nathan DM, Buse JB, Davidson MB, Ferrannini E, Holman RR, Sherwin R, Zinman B: Medical management of hyperglycemia in type 2 diabetes: a consensus algorithm for the initiation and adjustment of therapy: a consensus statement of the American Diabetes Association and the European Association for the Study of Diabetes. Diabetes Care 2009, 32:193-203

3. Ahren B, Schweizer A, Dejager S, Dunning BE, Nilsson PM, Persson M, Foley $J E$ : Vildagliptin enhances islet responsiveness to both hyper- and hypoglycemia in patients with type 2 diabetes. J Clin Endocrinol Metab 2009, 94:1236-1243.

4. Fonseca V, Schweizer A, Albrecht D, Baron MA, Chang I, Dejager S: Addition of vildagliptin to insulin improves glycaemic control in type 2 diabetes. Diabetologia 2007, 50:1148-1155.

5. Arnolds S, Dellweg S, Clair J, Dain MP, Nauck MA, Rave K, Kapitza C: Further improvement in postprandial glucose control with addition of exenatide or sitagliptin to combination therapy with insulin glargine and metformin: a proof-of-concept study. Diabetes Care 2010, 33:1509-1515.

6. Barnett AH, Charbonnel B, Donovan M, Fleming D, Chen R: Effect of saxagliptin as add-on therapy in patients with poorly controlled type 2 diabetes on insulin alone or insulin combined with metformin. Curr Med Res Opin 2012, 28:513-523.

7. Charbonnel B, Schweizer A, Dejager S: Combination therapy with DPP-4 inhibitors and insulin in patients with type 2 diabetes mellitus: what is the evidence? Hosp Pract (1995) 2013, 41:93-107.

8. Fonseca V, Baron M, Shao Q, Dejager S: Sustained efficacy and reduced hypoglycemia during one year of treatment with vildagliptin added to insulin in patients with type 2 diabetes mellitus. Horm Metab Res 2008, 40:427-430.

9. Hong ES, Khang AR, Yoon JW, Kang SM, Choi SH, Park KS, Jang HC, Shin H, Walford GA, Lim S: Comparison between sitagliptin as add-on therapy to insulin and insulin dose-increase therapy in uncontrolled Korean type 2 diabetes: CSI study. Diabetes Obes Metab 2012, 14:795-802.

10. Kothny W, Foley J, Kozlovski P, Shao Q, Gallwitz B, Lukashevich V: Improved glycaemic control with vildagliptin added to insulin, with or without metformin, in patients with type 2 diabetes mellitus. Diabetes Obes Metab 2013, 15(3):252-257

11. Lukashevich V, Schweizer A, Foley JE, Dickinson S, Groop PH, Kothny W: Efficacy of vildagliptin in combination with insulin in patients with type 2 diabetes and severe renal impairment. Vasc Health Risk Manag 2013, 9:21-28.

12. Rosenstock J, Rendell MS, Gross JL, Fleck PR, Wilson CA, Mekki Q: Alogliptin added to insulin therapy in patients with type 2 diabetes reduces $\mathrm{HbA}$ (1C) without causing weight gain or increased hypoglycaemia. Diabetes Obes Metab 2009, 11:1145-1152.

13. Schweizer A, Foley JE, Kothny W, Ahren B: Clinical evidence and mechanistic basis for vildagliptin's effect in combination with insulin. Vasc Health Risk Manag 2013, 9:57-64.

14. Vilsboll T, Rosenstock J, Yki-Jarvinen H, Cefalu WT, Chen Y, Luo E, Musser B Andryuk PJ, Ling Y, Kaufman KD, Amatruda JM, Engel SS, Katz L: Efficacy and safety of sitagliptin when added to insulin therapy in patients with type 2 diabetes. Diabetes Obes Metab 2010, 12:167-177.

15. Currie CJ, Johnson JA: The safety profile of exogenous insulin in people with type 2 diabetes: justification for concern. Diabetes Obes Metab 2012, $14: 1-4$.

16. Inzucchi SE, Bergenstal RM, Buse JB, Diamant M, Ferrannini E, Nauck M, Peters AL, Tsapas A, Wender R, Matthews DR, American Diabetes Association (ADA), European Association for the Study of Diabetes (EASD): Management of hyperglycaemia in type 2 diabetes: a patient-centered approach. Position statement of the American Diabetes Association (ADA) and the European Association for the Study of Diabetes (EASD). Diabetologia 2012, 55:1577-1596.

17. Mori Y, Taniguchi Y, Miyazaki S, Yokoyama J, Utsunomiya K: Effects of add-on treatment with sitagliptin on narrowing the range of glucose fluctuations in Japanese type 2 diabetes patients receiving insulin therapy. Diabetes Technol Ther 2013, 15:237-240.

18. Shimoda S, Iwashita S, Ichimori S, Matsuo Y, Goto R, Maeda T, Matsuo T, Sekigami T, Kawashima J, Kondo T, Matsumura T, Motoshima H, Furukawa $\mathrm{N}$, Nishida K, Araki E: Efficacy and safety of sitagliptin as add-on therapy on glycemic control and blood glucose fluctuation in Japanese type 2 diabetes subjects ongoing with multiple daily insulin injections therapy. Endocr J 2013, 60(10):1207-1214.

19. Van Genugten RE, Moller-Goede DL, Van Raalte DH, Diamant M: ExtraPancreatic Effects of Incretin-Based Therapies: Potential Benefit for Cardiovascular Risk Management in Type 2 Diabetes. Diabetes Obes Metab 2013, 15(7):593-606.

20. Davies M, Storms F, Shutler S, Bianchi-Biscay M, Gomis R: Improvement of glycemic control in subjects with poorly controlled type 2 diabetes: comparison of two treatment algorithms using insulin glargine. Diabetes Care 2005, 28:1282-1288.

21. Greven WL, Beulens JW, Biesma DH, Faiz S, De Valk HW: Glycemic variability in inadequately controlled type 1 diabetes and type 2 diabetes on intensive insulin therapy: a cross-sectional, observational study. Diabetes Technol Ther 2010, 12:695-699.

22. Meerwaldt R, Graaff R, Oomen PH, Links TP, Jager JJ, Alderson NL, Thorpe SR, Baynes JW, Gans RO, Smit AJ: Simple non-invasive assessment of advanced glycation endproduct accumulation. Diabetologia 2004, 47:1324-1330.

23. Meerwaldt R, Links T, Graaff R, Thorpe SR, Baynes JW, Hartog J, Gans R, Smit A: Simple noninvasive measurement of skin autofluorescence. Ann N Y Acad Sci 2005, 1043:290-298.

24. Esposito K, Ciotola M, Maiorino Ml, Gualdiero R, Schisano B, Ceriello A, Beneduce F, Feola G, Giugliano D: Addition of neutral protamine lispro insulin or insulin glargine to oral type 2 diabetes regimens for patients with suboptimal glycemic control: a randomized trial. Ann Intern Med 2008, 149:531-539.

25. Kooy A, De JJ, Lehert P, Bets D, Wulffele MG, Donker AJ, Stehouwer CD: Long-term effects of metformin on metabolism and microvascular and macrovascular disease in patients with type 2 diabetes mellitus. Arch Intern Med 2009, 169:616-625.

\section{doi:10.1186/1756-0500-7-579}

Cite this article as: de Ranitz-Greven et al:: Patients with Type 2 Diabetes Mellitus failing on oral agents and starting once daily insulin regimen; a small randomized study investigating effects of adding vildagliptin. BMC Research Notes 2014 7:579. 